\begin{tabular}{|l|l|l||}
\hline \multicolumn{2}{|c|}{ PublisherInfo } \\
\hline \hline PublisherName & $:$ & BioMed Central \\
\hline \hline PublisherLocation & $:$ & London \\
\hline \hline PublisherImprintName & $:$ & BioMed Central \\
\hline \hline
\end{tabular}

\title{
Oldest archaea?
}

\begin{tabular}{|l|l|l||}
\hline \multicolumn{2}{|c||}{ ArticleInfo } \\
\hline \hline ArticleID & $:$ & 3665 \\
\hline \hline ArticleDOI & $:$ & $10.1186 /$ gb-2000-1-6-reports0076 \\
\hline \hline ArticleCitationID & $:$ & reports0076 \\
\hline \hline ArticleSequenceNumber & $:$ & 15 \\
\hline \hline ArticleCategory & $:$ & Paper report \\
\hline \hline ArticleFirstPage & $:$ & 1 \\
\hline \hline ArticleLastPage & $:$ & 4 \\
\hline \hline & & RegistrationDate : 2000-9-20 \\
ArticleHistory & $:$ & Received \\
\hline ArticleCopyright & $:$ 2000-9-20 \\
\hline \hline ArticleGrants & $:$ & OnlineDate \\
\hline \hline ArticleContext & $:$ & 130591166 \\
\hline \hline
\end{tabular}




\section{James Cotton}

\section{Abstract}

A new division of the Archaea could be the most ancient living lineage.

\section{Significance and context}

Twenty years ago, oligonucleotide cataloguing supplied the first evidence that several groups of unusual microbes actually represented a wholly distinctive experiment in unicellular existence - the Archaebacteria or Archaea. The unusual biochemistry of these organisms provided evidence of shared ancestry, rather than convergent adaptation to their unusual lifestyles. All of the archaea known at the time occupied extreme environments, being extreme thermophiles, extreme halophiles, or both, or were ekeing out an existence by virtue of a clever metabolic trick - producing methane in strictly anaerobic conditions. The prevailing view seemed to be that the archaea were hanging on in a few specialized niches where the more successful eubacteria had failed to thrive - presumably helped by the unusual lipids unique to the group. In 1987, Carl Woese, in a much-cited and influential review, had "a growing feeling the three basic and highly unique phenotypes (of archaea)...are all that exist in the kingdom". That feeling has surely changed.

The existence of new uncultured types of archaea was first suggested in 1992, when two separate studies revealed archaea-like small-subunit (SSU) rRNA sequences in two different samples of seawater. These sequences were related to both the Crenarchaeota and Euryarchaeota, the two known lineages of archaea. Further work confirmed that these sequences represented organisms that were native to, and abundant in, cold seawater. Since this discovery, novel lineages of both archaean groups have been identified in a wide variety of marine, freshwater and terrestrial environments, confirming that the cultured archaea represent only a fraction of the group's diversity. Another surprise was to come, however, when Norman Pace and colleagues reported some particularly unusual archaeal sequences from a hot spring in Yellowstone National Park. These sequences were difficult to place phylogenetically, even when full-length SSU rRNA sequences became available, and are either very deeply branching crenarchaeotes, or an entirely new division of archaea (which they called 'korarchaeota'), or even a sister group to the Eukaryota. Using similar techniques, Kim et al. have now identified another branch of archaean diversity, even more ancient than the korarchaeote lineage. 


\section{Key results}

Kim et al. have twice previously reported unusual archaeal SSU rRNA sequences from diversity analyses of paddy-field soil archaeal communities. They have now designed PCR primers specific for these sequences, revealing the presence of similar molecules in paddy soils from around Japan. Using standard techniques, the researchers failed to produce amplification products using any primer pairs more than 700 base pairs apart, but succeeded in cloning 10 samples of around 645 nucleotides long. Phylogenies produced using a variety of different methods place these 10 sequences at the base of the Archaea (see Figure 1), below the Korarchaeota (which appear as basal crenarchaeotes in all of these analyses). The failure to detect these sequences in other more aerobic terrestrial soils strongly suggests that these organisms are at least facultative anaerobes.

Figure 1 Phylogenetic relationships of archaeal groups.

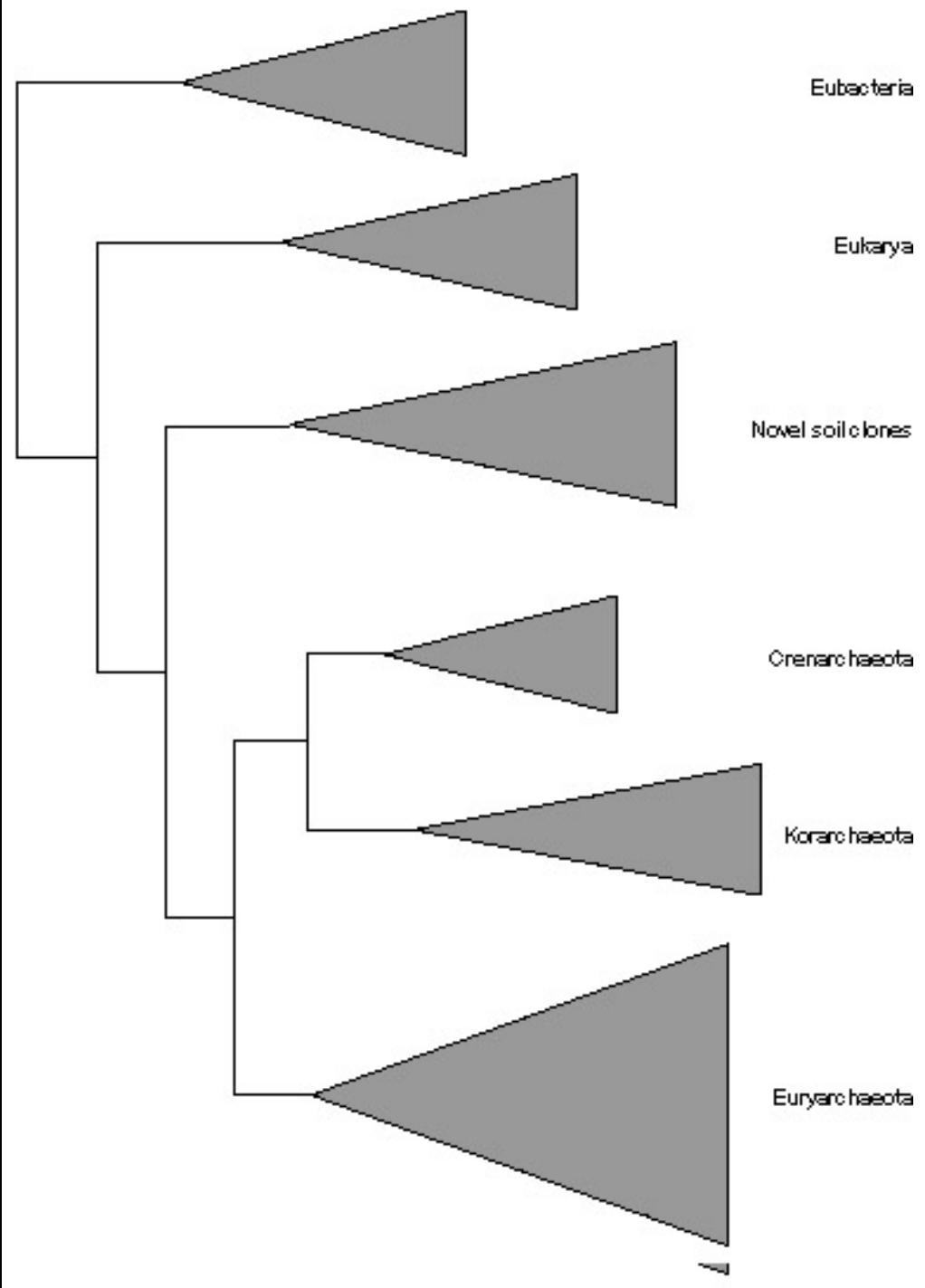




\title{
Conclusions
}

The sequences reported are likely to represent the first evidence of a major new lineage of the Archaea.

\section{Reporter's comments}

The short rRNA sequences this paper reports appear to be genuine - they fold into sensible secondary structures, and appear not to be chimeras of any other known RNAs. But there is a little more doubt about whether they truly represent organisms forming such a deep archaeal lineage. One very encouraging sign is that these new sequences consistently appear as basal to the Korarchaeota, which have previously been claimed to be the most basal archaea, and the position of the groups is stable across a number of different phylogenetic analyses. Bootstrap support is fairly low (between 60 and $70 \%$ in the different trees presented), but this would be expected for a deep branch in a SSU rRNA phylogeny. More sophisticated phylogenetic methods, such as spectral analysis, should make it clear how much support there is for other positions of the new clade on the tree. Ultimately, however, more data may be needed to increase the certainty with which we can place these organisms - a difficult problem in a field that has focused almost exclusively on rRNA genes.

The phylogenetic position of these prokaryotes and the korarchaeotes makes it most likely that the most ancient archaea alive today are mesophiles. The fact that the ancient crenarchaeotes are extreme thermophiles has been taken as evidence that life on Earth originated in extreme conditions, perhaps near hydrothermal vents, but there seems little evidence for this idea now. These newly identified bacteria would also seem to deal a fatal blow to the 'eocyte' theory of a sister-group relationship between the Crenarchaeota and eukaryotes. Most importantly, however, these new sequences remind us of our ignorance of the Earth's biota, and of the sheer diversity of living things. While we know a great deal about a relatively few microorganisms, we know next to nothing about most of the microbes that have been described; and we know literally nothing, except a few hundred base pairs of DNA sequence, about what is probably the vast majority of prokaryotes, both eubacterial and archaeal. With the importance of horizontal gene transfer becoming increasingly clear, our ignorance of these organisms means we cannot claim to know a great deal about the potential gene pool available to prokaryotes.

\section{Table of links}

\author{
Microbiology
}

\section{References}

1. Kim H, Honda D, Hanada S, Kanamori N, Shibata S, Miyaki T, Nakamura K, Oyaizu H: A deeply branched novel phylotype found in Japanese paddy soils. Microbiology. 2000, 146: 2309-2315. $1350-0872$ 